\title{
Comparing the Performance of Popular MEG/EEG Artifact Correction Methods in an Evoked-Response Study
}

\author{
Niels Trusbak Haumann, ${ }^{1}$ Lauri Parkkonen, ${ }^{2}$ Marina Kliuchko, ${ }^{3,4}$ \\ Peter Vuust, ${ }^{1}$ and Elvira Brattico ${ }^{1}$ \\ ${ }^{1}$ Center for Music in the Brain, Department of Clinical Medicine, Aarhus University and Royal Academy of Music, Aarhus/Aalborg, \\ Nørrebrogade 44, Building 10G, 4th and 5th floor, 8000 Aarhus C, Denmark \\ ${ }^{2}$ Department of Neuroscience and Biomedical Engineering, Aalto University School of Science, P.O. Box 12200, 00076 Aalto, Finland \\ ${ }^{3}$ Cognitive Brain Research Unit, Institute of Behavioural Sciences, University of Helsinki, P.O. Box 9 (Siltavuorenpenger 1 B), \\ 00014 Helsinki, Finland \\ ${ }^{4}$ BioMag Laboratory, HUS Medical Imaging Center, University of Helsinki and Helsinki University Hospital, P.O. Box 340, \\ Hospital District of Helsinki and Uusimaa, 00029 Helsinki, Finland
}

Correspondence should be addressed to Niels Trusbak Haumann; niels.haumann@clin.au.dk

Received 29 April 2016; Accepted 23 June 2016

Academic Editor: Victor H. C. de Albuquerque

Copyright (C) 2016 Niels Trusbak Haumann et al. This is an open access article distributed under the Creative Commons Attribution License, which permits unrestricted use, distribution, and reproduction in any medium, provided the original work is properly cited.

\begin{abstract}
We here compared results achieved by applying popular methods for reducing artifacts in magnetoencephalography (MEG) and electroencephalography (EEG) recordings of the auditory evoked Mismatch Negativity (MMN) responses in healthy adult subjects. We compared the Signal Space Separation (SSS) and temporal SSS (tSSS) methods for reducing noise from external and nearby sources. Our results showed that tSSS reduces the interference level more reliably than plain SSS, particularly for MEG gradiometers, also for healthy subjects not wearing strongly interfering magnetic material. Therefore, tSSS is recommended over SSS. Furthermore, we found that better artifact correction is achieved by applying Independent Component Analysis (ICA) in comparison to Signal Space Projection (SSP). Although SSP reduces the baseline noise level more than ICA, SSP also significantly reduces the signal-slightly more than it reduces the artifacts interfering with the signal. However, ICA also adds noise, or correction errors, to the waveform when the signal-to-noise ratio (SNR) in the original data is relatively low-in particular to EEG and to MEG magnetometer data. In conclusion, ICA is recommended over SSP, but one should be careful when applying ICA to reduce artifacts on neurophysiological data with relatively low SNR.
\end{abstract}

\section{Introduction}

Recordings of evoked-responses (also known as eventrelated potentials, ERPs, or event-related fields, ERFs) with electroencephalography (EEG) or magnetoencephalography (MEG) are widely used methods in cognitive and clinical neuroscience. One of the major challenges in research and clinical applications of evoked-responses is the prevalent strongly interfering electromagnetic signals from external objects and devices in the surrounding MEG or EEG measurement environment as well as nearby mechanical and biological electromagnetic sources originating from the head and other parts of the body of the subject. Since the interfering environmental noise from, for example, laboratory mechanics and electronic devices may be several orders of magnitude stronger than the brain signals of interest (for a review, see, e.g., [1]), it is necessary to remove this noise from the recordings during or after the measurements. Moreover, nonencephalic electromagnetic activity, such as that from the eyes and from the cardiac and facial muscles, is also recorded by EEG or MEG and can be up to a thousand times stronger than the encephalic signal of interest [1]. Since some of these interfering artifactual signals can be synchronous with the brain signal of interest, significant parts of the continuous measurement can be contaminated by artifacts. Hence, to ensure a reliable measurement, it is necessary, in addition to applying an average measure of an evoked-response across 
multiple time-locked data segments, also to omit or correct the data contaminated with artifacts.

In the clinical routine, data from patients having a limited control of muscular activity (such as stroke or dementia patients or preterm infants) or with ferromagnetic implants (such as cochlear implantees) typically contain a considerable amount of artifacts. The time constraints of experiments and tests on clinical populations exclude the possibility of a large number of trials that would allow discarding the artefactual ones. A viable alternative to simply rejecting parts of the recorded data is that of correcting the data. Both in clinical patient recordings and in experimental settings with healthy subjects, strong electromagnetic noise from electronic devices, static electricity, and in particular with regard to EEG also the $50 / 60-\mathrm{Hz}$ power-line noise may interfere significantly with the measurements [2]. When recording EEG in conjunction with transcranial magnetic stimulation (TMS), methods have been developed for reducing the strong TMS artifacts appearing in the recording [3]. In other cases where two neuroimaging modalities are employed simultaneously, special care must also be taken to reduce artifacts originating from the other modality. For recording EEG concurrently with functional magnetic resonance imaging ( $\mathrm{fMRI}$ ), it is necessary to reduce both imaging artifacts caused by the switching gradient fields [4] and ballistocardiogram artifacts caused by the subjects heart beats moving the skin and electrodes in relation to the strong magnetic field within the MRI scanner [5]. Furthermore, in combined EEG/MEG recordings one should be aware that eddy currents in the electrodes induce magnetic fields, which may introduce artifacts in the MEG recordings for signals in higher frequency ranges; however, signals at frequencies below $100 \mathrm{~Hz}$ are not critically affected by these types of artifacts [6].

Apart from external artifact sources, it is important to reduce the influence of the internal artifacts originating from the head and the rest of the body of the subject. Typically, MEG and EEG recordings are contaminated by relatively strong artifacts caused by the eyes [2, 7-9]. They can either be eye blinks (picked up mostly by the vertical EOG) contaminating particularly the lower frequencies or be saccades (visible mostly in horizontal EOG) also interfering at higher frequency ranges, where certain saccadic spike artifacts resemble high-frequency muscular artifacts [9]. Another typically interfering internal artifact is due to the electric activity of the cardiac muscle, measured by electrocardiography (ECG or EKG) $[2,10]$. Also, noises from different types of muscular activity, seen in electromyographic (EMG) signals, are also a typical issue in MEG and EEG recordings $[2,11]$. These muscular artifacts may be caused by mastication (chewing), deglutition (tongue movement), and respiration [2].

Different methods have been developed to reduce the influence of externally and internally originating artifacts. The externally originating interference can be minimized by applying physical shielding techniques in the laboratory [12], by using gradiometer sensors instead of magnetometers, by subtracting measurements of the external noise signals recorded by one or more reference sensors or by applying online or offline spatial filtering methods. MEG systems by Elekta Oy (Helsinki, Finland) comprise both magnetometers and gradiometers and they employ spatial filtering techniques such as Signal Space Projection (SSP) and Signal Space Separation (SSS) and its temporal extension (tSSS) implemented in the Elekta Neuromag ${ }^{\circledR}$ MaxFilter $^{\mathrm{TM}}$ software [13]. The SSS method is based on Maxwell equations and the multichannel measurement of the magnetic field distribution; by using a basis comprising spherical harmonic functions, contributions of signal sources within the sensor array (brain signals) can be separated from sources external to the array [13-18]. Since SSS is purely a spatial filter, which only reduces noise originating from the external sources, it retains also those brain signals that oscillate at the same frequency as an external noise source [14]. However, nearby sources of artifacts caused by, for example, movement of magnetic materials, such as dental braces or implants, cannot be sufficiently reduced by applying SSS alone.

The tSSS method is additionally able to filter out interferences from artifactual sources in the space between the brain and the MEG sensor array, by reducing signals in the common subspace through comparisons of the time series in the internal and external spaces. For instance, it has been shown that tSSS makes it possible to locate brain sources on the cortex with beamformer methods in clinical patients, although these patients wore strongly magnetically interfering dental braces; thereby, tSSS seems to allow extending the clinical population compatible with MEG [17].

The internally originating artifacts can be reduced by applying band-pass filtering [19] and component analysis such as Principal Component Analysis (PCA) or Independent Component Analysis (ICA) [20-43] or by recording the artifacts to be removed, identifying their contribution to the data by means of linear regression and subtracting them out [10,44, 45]. Also, methods for ignoring the artifactual sources have been implemented as part of source analysis algorithms [46, 47]. With regard to the component analysis approaches, PCA is applied to estimate components explaining the highest variance in the data, such as strong artifacts. ICA is able to estimate components that explain variance originating from statistically independent sources, thereby reducing the risk of including signals of interest in the derived artifact components. The ICA algorithms, and, in particular, the infomax version of ICA, have gained popularity as an efficient method for separating the recorded signals into statistically independent components [43]. By inspecting the independent components, only the artifactual components can be rejected to reduce the influence of the artifacts on the data. An alternative method of Signal Space Projection (SSP) has gained some popularity in open source software packages $[48,49]$. SSP also decomposes the data into components, often based on a prior PCA; however, in contrast with ICA, these components may not be statistically independent, and therefore there is a risk that artifactual and brain signals of interest may be reduced simultaneously [48].

To investigate the performances and risks of using different popular artifact correction methods, we compared here results achieved by applying SSS, tSSS, ICA, and SSP. We 
chose to study the performance of the correction methods on the Mismatch Negativity (MMN) response, which is a well-known evoked-response [50, 51]. In particular, we wanted to investigate (1) whether tSSS improves the data quality in healthy subjects not wearing any magnetically disturbing implants; (2) whether the faster SSS alternative performs as well as the more computationally demanding tSSS; and (3) whether ICA is preferable over SSP (or vice versa) for reduction of typical artifacts in healthy subjects.

\section{Methods}

2.1. Participants. A sample of ten volunteers from a larger database named "Tunteet" was chosen (for a description of the experimental protocol, see Kliuchko et al., submitted). The participants were six females and four males. Three participants were nonmusicians, three were amateur musicians, and four were professional musicians. All participants were right-handed, and their average age was 24.8 years (range 1835 years). Written informed consent was obtained from each participant, and the study was approved by the local ethics committee.

2.2. Experimental Paradigm. The participants listened to a melody pattern of $2100 \mathrm{~ms}$, repeated with variations during $\sim 25$ minutes. The melody patterns were created from digital piano tones (McGill University Master Samples) and followed the rules of Western tonal music. All melodies started with a triad $(300 \mathrm{~ms})$ followed by four single tones (two of $125 \mathrm{~ms}$ and two of $300 \mathrm{~ms}$ ) and an ending tone $(575 \mathrm{~ms}$ ) all separated by $50 \mathrm{~ms}$ silent gap. Between all melodies there was a silent gap of $125 \mathrm{~ms}$. Deviations of six types were inserted into the melody patterns to evoke MMN responses to a deviant tone as compared with corresponding unaltered standard. The deviants are explained in Table 1 [52-54].

In total, the tested sample contained 120 brain responses, which comprised the responses to the six standard and six deviant conditions from each of the ten participants.

2.3. Data Acquisition. The simultaneous MEG and EEG data were collected at the BioMag Laboratory of the Helsinki University Central Hospital. The measurements were performed in an electrically and magnetically shielded room (ETSLindgren Euroshield, Eura, Finland) with Vectorview ${ }^{\mathrm{TM}} 306-$ channel MEG system (Elekta Neuromag, Elekta Oy, Helsinki, Finland) equipped with a compatible EEG system. The MEG system had 102 triple-sensor elements, each comprising two orthogonal planar gradiometers and one magnetometer. A 64-channel EEG electrode cap was used. The reference electrode was placed on the nose tip and the ground electrode was on the right cheek. Blinks, as well as vertical and horizontal eye movements, were measured with four electrodes attached above and below the left eye and close to the external eye corners on both sides. Four head position indicator coils were placed on top of the EEG cap. Their positions were determined with respect to the nasion and preauricular points by an Isotrak 3D digitizer (Polhemus, Colchester, VT,
USA). MEG and EEG data were recorded with a sample rate of $600 \mathrm{~Hz}$.

During the measurement, subjects were comfortably seated and watched a silenced movie with subtitles. The stimuli were presented with Presentation software (Neurobehavioral Systems, Ltd.). The sound was delivered through a pair of pneumatic headphones at individually adjusted loudness.

2.4. Artifact Correction. Elekta Neuromag MaxFilter 2.2 Signal Space Separation (SSS) and temporal Signal Space Separation (tSSS) $[13,16]$ were applied separately to compare their individual performance. For both SSS and tSSS, we used the default inside expansion order of 8 , outside expansion order of 3, automatic optimization of both inside and outside bases, and automatic detection and correction of bad MEG channels. Additionally, for both SSS and tSSS the specific fine calibration and cross talk correction data for the recording site and date were applied. For the tSSS, we used the default subspace correlation limit of 0.980 and raw data buffer length of 10 seconds. The spatially filtered data were saved in 32-bit float format at a sampling rate of $600 \mathrm{~Hz}$.

Correction for internal artifacts with Signal Space Projection (SSP) was performed with the MNE Python version 0.11.0 released with the MNE software version 2.7.4-3485 $[55,56]$. We applied the default automatic settings, where two principal components per artifact type are detected for eye artifacts and for cardiac artifacts. Subsequently, the detected ocular and cardiac artifact component projections were removed from the data.

Independent Component Analysis- (ICA-) based correction for internal artifacts was achieved by applying the logistic infomax algorithm implemented in the runica function [57] for MATLAB $^{\circledR}$ (MathWorks, Natick, Massachusetts). First, the data were reduced to 64 principal components. The independent components were then estimated for the EEG channels, MEG magnetometers, and MEG planar gradiometers separately. The resulting components were inspected, and one component projection per vertical eye movement, horizontal eye movement, or cardiac artifact type (explaining most variance) was removed from the data, when the artifact component was observed. On average, the total number of observed artifact components per subject was 1.7 (1-2) for the EEG, 1.8 (1-3) for the MEG magnetometers, and 2.0 (1-3) for the MEG gradiometers.

2.5. Data Analysis. Event-related EEG and MEG responses were extracted as single-trial epochs with a time window of 0 to $400 \mathrm{~ms}$ after the standard or deviant tone onset. The trials were baseline-corrected by applying a baseline of -100 to $0 \mathrm{~ms}$ before the tone onset. Since the planar gradiometer sensors measure along two orthogonal directions, the data from each pair of longitudinal and latitudinal sensors were combined by applying the Pythagorean distance formula, as implemented in the FieldTrip toolbox for MATLAB (Donders Institute for Brain, Cognition and Behaviour/Max Planck Institute, Nijmegen, Netherlands) $[58] ; d=\sqrt{\text { longitudinal }^{2}+\text { latitudinal }^{2}}$. 
TABLE 1: Deviant tones. Type of deviant is the type of change applied to the tone, and description explains the change. Occurrence describes whether the change is presented in different patterns randomly (single) or is present constantly in each following pattern from the first presentation until the next deviant of the same type occurs (consistent). Tone number denotes at which tone of the melody pattern the change may occur. Melodies with deviants column shows the percentage of melodies containing the particular type of deviant.

\begin{tabular}{|c|c|c|c|c|}
\hline Type of deviant & Description & Occurrence & Tone number & $\begin{array}{c}\text { Melodies with } \\
\text { deviants }\end{array}$ \\
\hline Mistuning & $3 \%$ pitch frequency increase & Single & 1st, 2nd, 4th & $14 \%$ \\
\hline Timbre & Flute sound & Single & 1st, 3rd, 4th, ending & $8 \%$ \\
\hline Timing delay & $100 \mathrm{~ms}$ silent gap before the tone & Single & $\begin{array}{l}\text { 1st, } 2 \text { nd, 3rd, } \\
\text { ending }\end{array}$ & $8 \%$ \\
\hline Melody modulation & Tone replacement & Consistent & 3 rd, 4 th & $12 \%$ \\
\hline Rhythm modulation & $\begin{array}{l}\text { Duration switch between two successive } \\
\text { tones }\end{array}$ & Consistent & 2nd, 3rd & $7 \%$ \\
\hline Transposition & Semitone pitch change of a pattern & Consistent & Initial triad & $16 \%$ \\
\hline
\end{tabular}

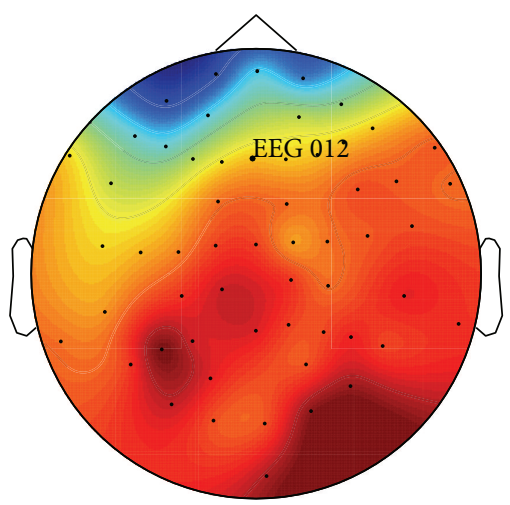

(a)

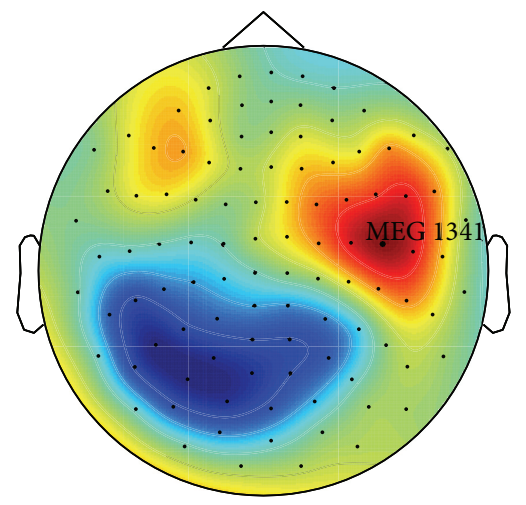

(b)

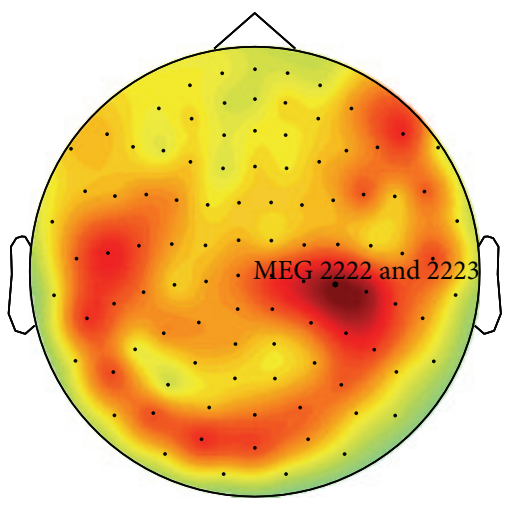

(c)

FIGURE 1: Topographical plots of MMN responses (without correction for internal artifacts). Showing topographical plots of MMN responses to the mistuning deviants, which elicited the strongest and most consistent MMN responses in the tested sample in the EEG (a), MEG magnetometers (b), and MEG gradiometers (c). The plots are based on grand averages from uncorrected data (only preprocessed with tSSS) by applying a time window from 125 to $155 \mathrm{~ms}$ after the tone onset.

For the sake of clarity, we performed the subsequent analyses on one channel of each sensor type, those in which the highest MMN amplitude was measured within a typical MMN latency range of 75-200 ms. In this case, we analyzed the event-related waveforms from EEG channel 012 (frontal site), magnetometer channel MEG 1341 (right temporal site), and the combined planar gradiometer channels MEG 2222 and 2223 (right temporal site) (see Figure 1). These analyzed channels behaved reliably and were not detected as bad channels or subjected to any additional correction.

We measured the MMN amplitude in response to each type of deviant tone by taking the average value across the time window from 125 to $155 \mathrm{~ms}$ after the tone onset. To compare the noise levels after utilizing each artifact correction method, we first used a baseline standard deviation measure. Since a flat baseline is desirable, we applied a baseline standard deviation (STD) measure to show the flatness of the baseline in a single trial (where lower baseline STD means a more flat baseline) [59]. We calculated the standard deviation across the baseline time points from -100 to $0 \mathrm{~ms}$ (in relation to the stimulus onset) in each trial separately and extracted the mean baseline STD across trials. Also, minimal variance in the measured signal across trials is desirable. Therefore, we also calculated the signal STD across trials for each time point in 125 to $155 \mathrm{~ms}$ :

$$
\sigma_{\bar{x}}=\sqrt{\frac{\sum_{i=1}^{n}\left(x_{i}-\bar{x}\right)^{2}}{N}},
$$

where $x$ is the measured value, $\bar{x}$ is the mean value, $i$ is the trial number, and $N$ is the total number of trials [18] and we averaged these values to obtain the mean signal STD. For additional comparisons, we applied a signal-to-noise ratio measure; $\mathrm{SNR}=$ amplitude $/ \sigma_{\bar{x}}[18]$.

The Mismatch Negativity (MMN) evoked-response is analyzed by comparing the average response to the deviant stimulus with the average response to the standard stimulus $[50,51]$. Also, MMN waveforms are conventionally calculated by subtracting the average response to the standard stimulus from the average response to the deviant stimulus $[50,51]$. However, we here analyze the noise levels across multiple single-trial MMN responses, which does not allow us to create difference waveforms by simply subtracting particular 


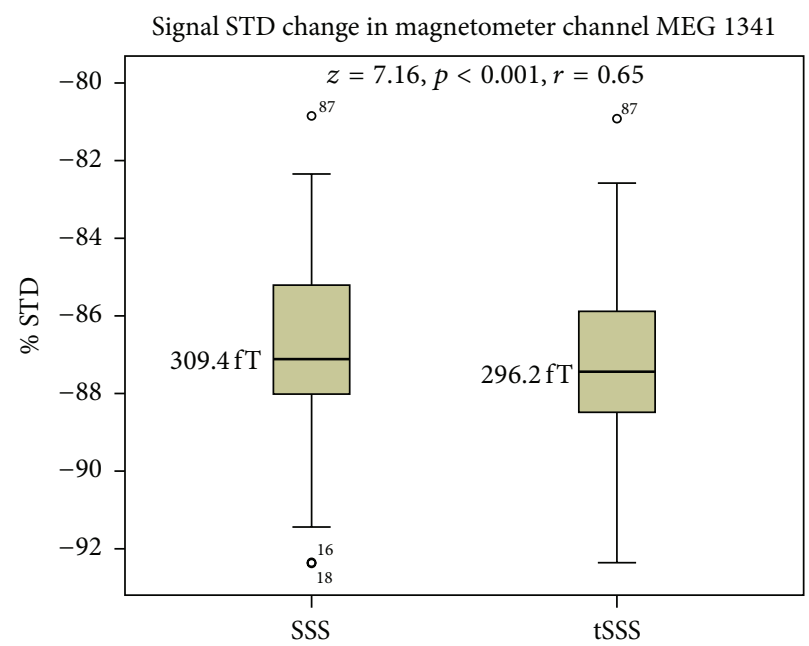

(a)

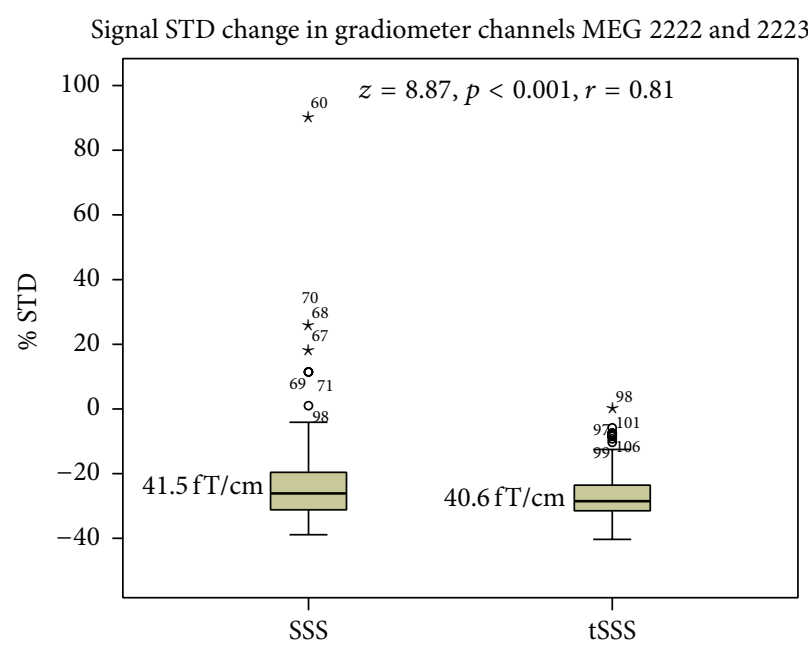

(b)

FIGURE 2: Percentagewise standard deviation (STD) reduction in the signal latency range achieved by applying the SSS or tSSS methods in comparison to the level (at 0\% STD) in the raw data recording without spatial filtering. The differences are shown for the amplitude peak channel of the magnetometers (a) and gradiometers (b) in Tukey box plots. Bold horizontal lines indicate medians, brown boxes indicate the first 50 percent of the case range, and vertical lines indicate cases within the 1.5 interquartile range from the edge of the $50 \%$ of the cases. Outliers more than 1.5 (circles) or 3.0 (stars) interquartile ranges from the edge of the $50 \%$ of the cases are denoted with circles or stars, and case numbers are provided for each outlier. The median values are shown in femtoteslas (fT) and femtoteslas per centimeter (fT/cm). $z$ and $r$ indicate the effect size, and $p$ indicates the level of statistical significance.

pairs of deviant and standard trials among multiple equally possible pairings of trials. The responses to both deviant and standard stimuli are relevant for any subsequent analyses of $\mathrm{MMN}$, and it is therefore important to know the noise levels of responses to both stimuli. Therefore, we here analyzed the noise levels in the responses to both the deviant and standard stimuli.

Statistical comparisons were made with SPSS version 20 (IBM, Armonk, New York, USA). Since the resulting values were not normally distributed, we applied the Wilcoxon signed-rank test to compare the values achieved after utilizing the different artifact correction methods.

\section{Results}

3.1. SSS and tSSS. A statistically significant and slightly better reduction of the signal standard deviation (STD) is achieved by applying tSSS in comparison to SSS for for both the MEG magnetometer and gradiometer data (see Figure 2). Importantly, in $6 \%(7 / 120)$ of the tested cases, the signal STD actually increases when applying SSS to the MEG gradiometer data, whereas the signal STD either is retained or decreases when the tSSS method is applied.

3.2. ICA and SSP. From grand average waveforms of the event-related responses (across all participants and conditions), it can be seen that the SSP-based artifact correction reduces the signal amplitude, whereas the signal amplitude is similar before and after the artifact correction based on ICA (see Figure 3).

The SSP method results in lower baseline standard deviation (STD) and signal STD in comparison to the ICA method (see Figure 4). The baseline STD even appears to increase when applying the ICA-based artifact correction, in particular with respect to the EEG and magnetometer channels. However, for the gradiometers, ICA yields slightly but statistically significantly better SNR than that achieved by applying the SSP method (see Figure 5).

The SNR achieved by applying the ICA-based artifact correction is similar to that achieved by applying the SSP method with respect to the EEG and magnetometer channels. However, for the gradiometers applying the ICA method results in statistically significantly and slightly better SNR than that achieved by applying the SSP method (see Figure 5).

\section{Discussion}

We compared the noise suppression results achieved with SSS and tSSS on healthy subjects not wearing magnetized material. MaxFilter with tSSS resulted in better suppression of artifacts from external and nearby noise sources in comparison to SSS. In particular, the application of tSSS instead of SSS was important with respect to the MEG gradiometers, since SSS correction in $6 \%$ of the cases resulted in an increase of the noise level in the MEG gradiometer data, and thus the reliability of the gradiometer data decreased in comparison to that before SSS. We also compared the performance of ICA and SSP in reducing internal electrophysiological artifacts, originating from eye movements and heart beats of the participants. The ICA-based artifact correction performed better than the SSP method. The SSP method reduced part of the signal of interest along with the artifacts, and the SNR was slightly higher after applying the ICA method than after applying the SSP method. However, after ICA-based artifact 

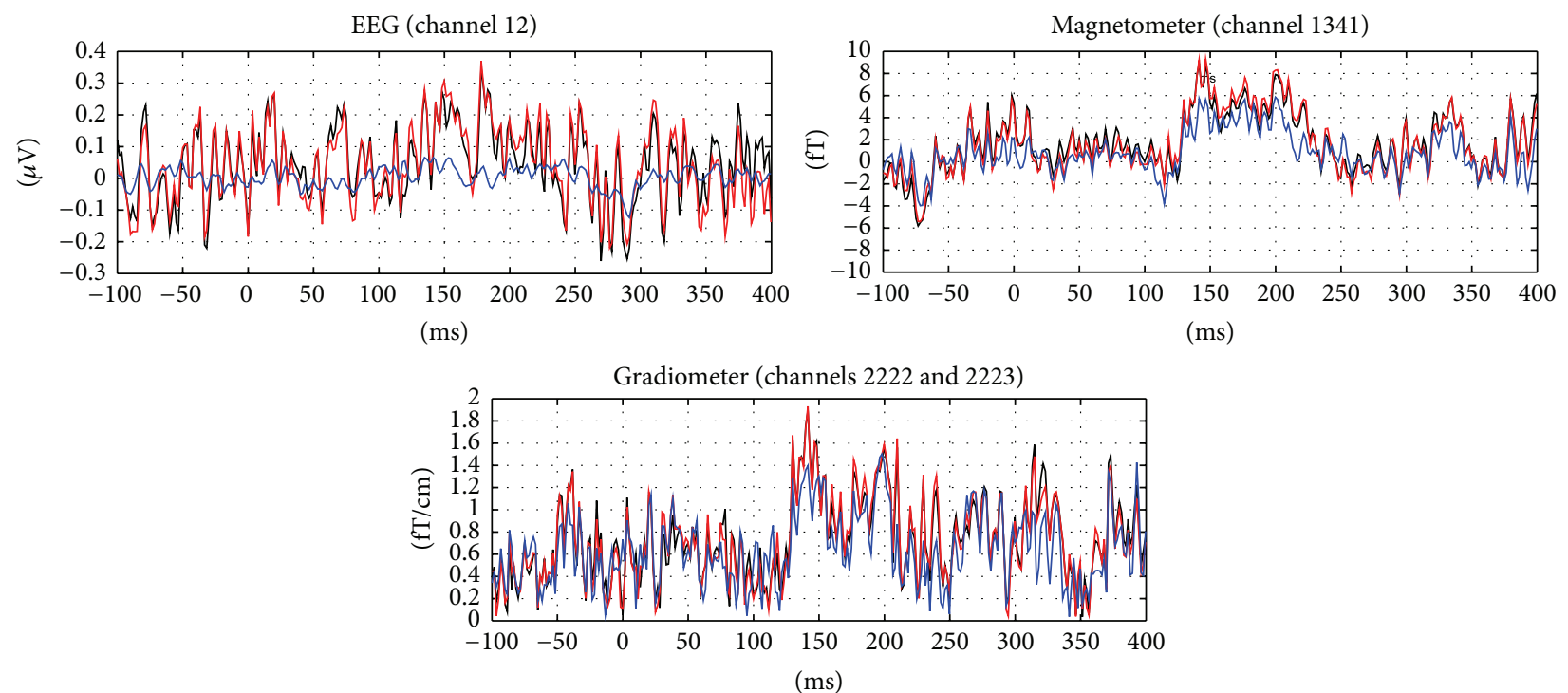

Grand average event-related waveforms

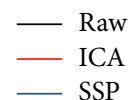

FIGURE 3: Grand average event-related waveforms. Showing the channels with the signal peak amplitude among the EEG channels, magnetometers, and gradiometers, without any artifact correction (black) and after artifact correction based on Independent Component Analysis (ICA) (red) and Signal Space Projection (SSP) (blue).

correction the baseline noise level increased, in particular in the EEG and magnetometer channels, which have relatively low SNR in the original data. These findings support both the importance of reducing the bias on measures of evokedresponses with EEG and MEG caused by artifacts and the importance of minimizing the bias introduced by errors in artifact correction methods.

With regard to the suppression of external noise, we here observed that the averaged MMN evoked-response in $6 \%$ of the cases with the MEG gradiometers even became more unreliable after applying SSS correction than before. Possibly, the influence of nearby artifacts on the evokedresponse can become stronger after the external artifacts have been reduced with the SSS. When tSSS is applied the influence of such nearby artifacts on the evoked-responses would be reduced. In general, it seems relevant to further investigate whether correction methods for reducing one type of artifact, such as SSS, in some cases might enhance the influence of other artifacts on the averaged event-related waveform.

The comparisons of the ICA and SSP methods for suppression of internal artifacts revealed particular biases appearing after the corrections. For the SSP method, there is a risk that the artifacts and signals of interest are not described by orthogonal components [48]. We observed this issue after applying the SSP artifact correction method, and we found that part of the signal of interest was reduced along with the influence of the artifacts. For the ICA method, there is another risk that after correction on channels with relatively low SNR is applied-such as correction on EEG channels, magnetometer channels, and channels located distantly from the signal peaks-additional noise is added to these channels. This happens because the errors in estimating the mixing matrix for the ICA will increase when the SNR decreases [60]. Our results emphasized this issue in showing that the baseline noise level increased after applying the ICA-based correction, in particular in the EEG channels and in the MEG magnetometer channels, and also the difference in SNR between applying ICA and SSP was smaller for the EEG channels and MEG magnetometer channels than for the MEG gradiometer channels, suggesting a relatively smaller improvement for the EEG and MEG magnetometer channels after applying the ICA-based artifact correction.

In summary, our test results suggest that tSSS is recommendable for reducing the influence of artifacts originating from external and nearby sources instead of SSS only. We find that the noise level decreases more with tSSS than with SSS in this sample of EEG and MEG data from healthy participants despite the fact that they were not wearing strongly magnetized materials. For the reduction of internal physiological artifacts, we showed that the highest signal-to-noise ratio (SNR) is achieved with ICA-based artifact correction on the tested sample. However, both ICA- and SSP-based artifact corrections are subject to certain limitations. In particular, one must be aware of the risk when processing data with relatively low SNR, such as EEG and MEG magnetometer data, that artifact correction based on ICA may decrease the interference from artifacts while simultaneously increasing the noise level, due to increasing errors in estimating the mixing matrix in the context of data with lower SNR levels. 

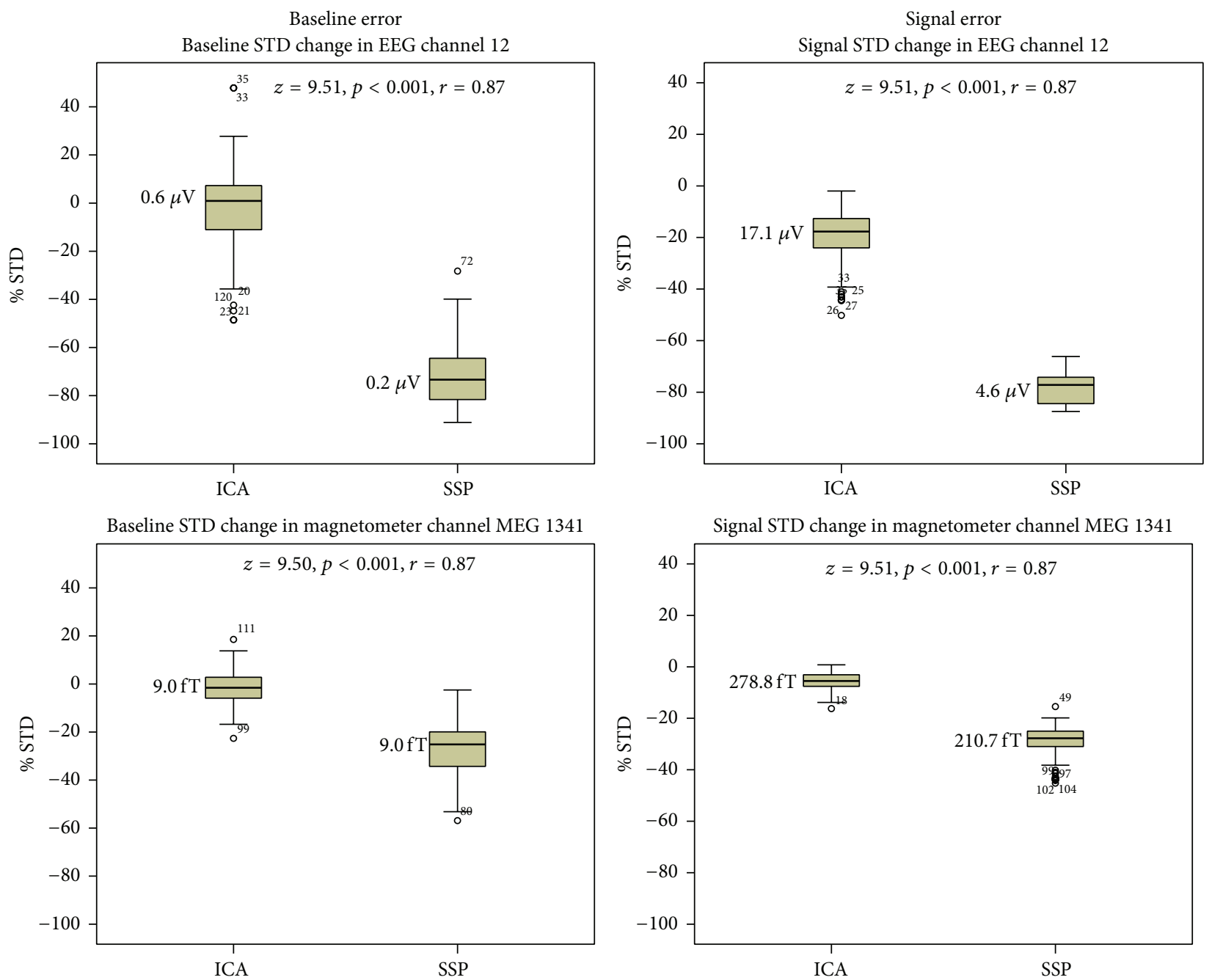

Baseline STD change in gradiometer channels MEG 2222 and 2223

Signal STD change in gradiometer channels MEG 2222 and 2223
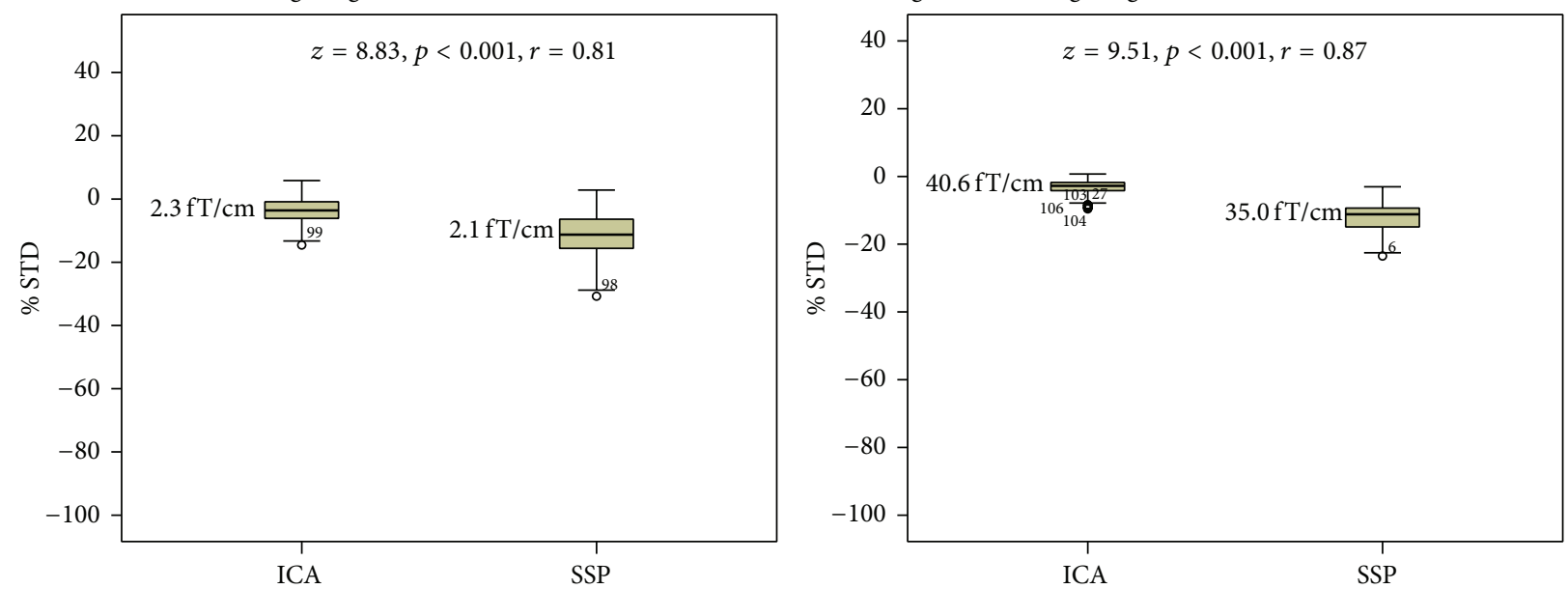

FIGURE 4: Percentagewise baseline standard deviation (STD) and signal STD reduction achieved by applying ICA or SSP methods in comparison to the level (at $0 \% \mathrm{STD}$ ) in the raw data recording with tSSS only. Median values are shown in microvolts $(\mu \mathrm{V})$, femtoteslas (fT), and femtoteslas per centimeter $(\mathrm{fT} / \mathrm{cm})$. Outliers more than 1.5 interquartile ranges from the edge of the $50 \%$ of the cases are denoted with circles. Case numbers are provided for each outlier. 


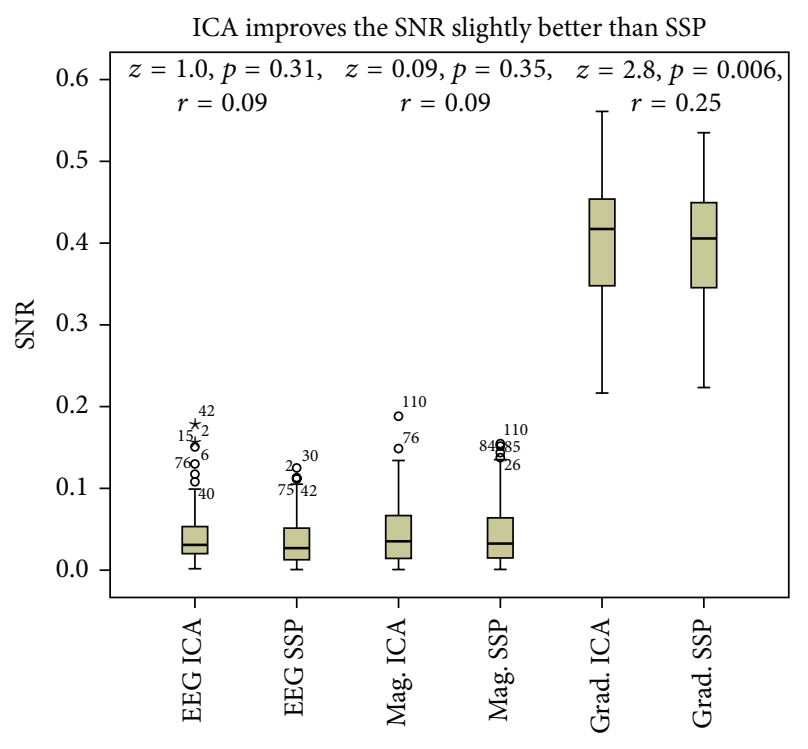

FIGURE 5: Signal-to-noise ratios (SNR) achieved by applying artifact correction based on Independent Component Analysis (ICA) or Signal Space Projection (SSP). Tukey box plot showing the SNR levels achieved by applying the ICA or SSP method in the signal amplitude peak channel of the EEG, magnetometers (Mag.), and gradiometers (Grad.) (the SNR shows the relationship between the signal and noise level in single trials). Outliers more than 1.5 (circles) or 3.0 (stars) interquartile ranges from the edge of the $50 \%$ of the cases are denoted with circles or stars, and case numbers are provided for each outlier.

\section{Competing Interests}

The authors declare that they have no competing interests.

\section{References}

[1] M. Hämäläinen, R. Hari, R. J. Ilmoniemi, J. Knuutila, and O. V. Lounasmaa, "Magnetoencephalography-theory, instrumentation, and applications to noninvasive studies of the working human brain," Reviews of Modern Physics, vol. 65, no. 2, pp. 413497, 1993.

[2] W. O. Tatum, B. A. Dworetzky, W. D. Freeman, and D. L. Schomer, "Artifact: recording EEG in special care units," Journal of Clinical Neurophysiology, vol. 28, no. 3, pp. 264-277, 2011.

[3] F. Morbidi, A. Garulli, D. Prattichizzo, C. Rizzo, and S. Rossi, "Application of Kalman filter to remove TMS-induced artifacts from EEG recordings," IEEE Transactions on Control Systems Technology, vol. 16, no. 6, pp. 1360-1366, 2008.

[4] M. Koskinen and N. Vartiainen, "Removal of imaging artifacts in EEG during simultaneous EEG/fMRI recording: reconstruction of a high-precision artifact template," NeuroImage, vol. 46, no. 1, pp. 160-167, 2009.

[5] X. Wan, K. Iwata, J. Riera, T. Ozaki, M. Kitamura, and R. Kawashima, "Artifact reduction for EEG/fMRI recording: nonlinear reduction of ballistocardiogram artifacts," Clinical Neurophysiology, vol. 117, no. 3, pp. 668-680, 2006.

[6] R. Murdick and B. J. Roth, "Magneto-encephalogram artifacts caused by electro-encephalogram electrodes," Medical and Biological Engineering and Computing, vol. 41, no. 2, pp. 203-205, 2003.
[7] R. J. M. Somsen and B. van Beek, "Ocular artifacts in children's EEG: selection is better than correction," Biological Psychology, vol. 48, no. 3, pp. 281-300, 1998.

[8] D. Hagemann and E. Naumann, "The effects of ocular artifacts on (lateralized) broadband power in the EEG," Clinical Neurophysiology, vol. 112, no. 2, pp. 215-231, 2001.

[9] C. Carl, A. Açık, P. König, A. K. Engel, and J. F. Hipp, “The saccadic spike artifact in MEG," NeuroImage, vol. 59, no. 2, pp. 1657-1667, 2012.

[10] I. Tal and M. Abeles, "Cleaning MEG artifacts using external cues," Journal of Neuroscience Methods, vol. 217, no. 1-2, pp. 3138, 2013.

[11] J. Ma, P. Tao, S. Bayram, and V. Svetnik, "Muscle artifacts in multichannel EEG: characteristics and reduction," Clinical Neurophysiology, vol. 123, no. 8, pp. 1676-1686, 2012.

[12] D. Cohen, U. Schläpfer, S. Ahlfors, M. Hämäläinen, and E. Halgren, New Six-Layer Magnetically-Shielded Room for MEG, 2002.

[13] S. Taulu and J. Simola, "Spatiotemporal signal space separation method for rejecting nearby interference in MEG measurements," Physics in Medicine and Biology, vol. 51, no. 7, pp. 17591768,2006

[14] T. Song, K. Gaa, L. Cui, L. Feffer, R. R. Lee, and M. Huang, "Evaluation of signal space separation via simulation," Medical and Biological Engineering and Computing, vol. 46, no. 9, pp. 923-932, 2008.

[15] T. Song, L. Cui, K. Gaa et al., "Signal space separation algorithm and its application on suppressing artifacts caused by vagus nerve stimulation for magnetoencephalography recordings," Journal of Clinical Neurophysiology, vol. 26, no. 6, pp. 392-400, 2009.

[16] S. Taulu and R. Hari, "Removal of magnetoencephalographic artifacts with temporal signal-space separation: demonstration with single-trial auditory-evoked responses," Human Brain Mapping, vol. 30, no. 5, pp. 1524-1534, 2009.

[17] A. Hillebrand, P. Fazio, J. C. De Munck, and B. W. Van Dijk, "Feasibility of clinical magnetoencephalography (MEG) functional mapping in the presence of dental artefacts," Clinical Neurophysiology, vol. 124, no. 1, pp. 107-113, 2013.

[18] A. Gonzalez-Moreno, S. Aurtenetxe, M.-E. Lopez-Garcia, F. del Pozo, F. Maestu, and A. Nevado, "Signal-to-noise ratio of the MEG signal after preprocessing," Journal of Neuroscience Methods, vol. 222, pp. 56-61, 2014.

[19] S. J. Schiff, A. Aldroubi, M. Unser, and S. Sato, "Fast wavelet transformation of EEG," Electroencephalography and Clinical Neurophysiology, vol. 91, no. 6, pp. 442-455, 1994.

[20] T.-P. Jung, C. Humphries, T.-W. Lee et al., "Removing electroencephalographic artifacts: comparison between ICA and PCA," in Proceedings of the IEEE Signal Processing Society Workshop Neural Networks for Signal Processing VIII, pp. 63-72, Cambridge, UK, August-September 1998.

[21] S. Ikeda and K. Toyama, "Independent component analysis for noisy data-MEG data analysis," Neural Networks, vol. 13, no. 10, pp. 1063-1074, 2000.

[22] R. Vigário, J. Särelä, V. Jousmiki, M. Hämäläinen, and E. Oja, "Independent component approach to the analysis of EEG and MEG recordings," IEEE Transactions on Biomedical Engineering, vol. 47, no. 5, pp. 589-593, 2000.

[23] J. Iriarte, E. Urrestarazu, M. Valencia et al., "Independent component analysis as a tool to eliminate artifacts in EEG: a quantitative study," Journal of Clinical Neurophysiology, vol. 20, no. 4, pp. 249-257, 2003. 
[24] C. J. James and O. J. Gibson, “Temporally constrained ICA: an application to artifact rejection in electromagnetic brain signal analysis," IEEE Transactions on Biomedical Engineering, vol. 50, no. 9, pp. 1108-1116, 2003.

[25] M. Kawakatsu, "Application of ICA to MEG noise reduction," in Proceedings of the Independent Component Analysis and Blind Signal Separation (ICA '03), pp. 535-541, Nara, Japan, April 2003.

[26] C. A. Joyce, I. F. Gorodnitsky, and M. Kutas, "Automatic removal of eye movement and blink artifacts from EEG data using blind component separation," Psychophysiology, vol. 41, no. 2, pp. 313325, 2004.

[27] Y. Konno, J. Cao, T. Takeda, H. Endo, T. Arai, and M. Tanaka, "Evaluation of brain source separation for MEG data applying JADE, fast-ICA and natural gradient-based algorithm with robust pre-whitening technique," Journal of Signal Processing, vol. 8, no. 6, pp. 461-472, 2004.

[28] A. C. Tang, M. T. Sutherland, and C. J. McKinney, "Validation of SOBI components from high-density EEG," NeuroImage, vol. 25, no. 2, pp. 539-553, 2005.

[29] W. De Clercq, A. Vergult, B. Vanrumste, W. Van Paesschen, and S. Van Huffel, "Canonical correlation analysis applied to remove muscle artifacts from the electroencephalogram," IEEE Transactions on Biomedical Engineering, vol. 53, no. 12, pp. 25832587, 2006.

[30] J. Escudero, R. Hornero, D. Abásolo, A. Fernández, and M. López-Coronado, "Artifact removal in magnetoencephalogram background activity with independent component analysis," IEEE Transactions on Biomedical Engineering, vol. 54, no. 11, pp. 1965-1973, 2007.

[31] S. P. Fitzgibbon, D. M. W. Powers, K. J. Pope, and C. R. Clark, "Removal of EEG noise and artifact using blind source separation," Journal of Clinical Neurophysiology, vol. 24, no. 3, pp. 232-243, 2007.

[32] J. Dammers, M. Schiek, F. Boers et al., "Integration of amplitude and phase statistics for complete artifact removal in independent components of neuromagnetic recordings," IEEE Transactions on Biomedical Engineering, vol. 55, no. 10, pp. 23532362, 2008.

[33] S. Hoffmann and M. Falkenstein, "The correction of eye blink artefacts in the EEG: a comparison of two prominent methods," PLoS ONE, vol. 3, no. 8, Article ID e3004, 2008.

[34] D. Mantini, R. Franciotti, G. L. Romani, and V. Pizzella, "Improving MEG source localizations: an automated method for complete artifact removal based on independent component analysis," NeuroImage, vol. 40, no. 1, pp. 160-173, 2008.

[35] J. Escudero, R. Hornero, D. Abásolo, and A. Fernández, "Quantitative evaluation of artifact removal in real magnetoencephalogram signals with blind source separation," Annals of Biomedical Engineering, vol. 39, no. 8, pp. 2274-2286, 2011.

[36] A. Hyvärinen, "Testing the ICA mixing matrix based on intersubject or inter-session consistency," NeuroImage, vol. 58, no. 1 , pp. 122-136, 2011.

[37] R. J. Korhonen, J. C. Hernandez-Pavon, J. Metsomaa, H. Mäki, R. J. Ilmoniemi, and J. Sarvas, "Removal of large muscle artifacts from transcranial magnetic stimulation-evoked EEG by independent component analysis," Medical \& Biological Engineering \& Computing, vol. 49, no. 4, pp. 397-407, 2011.

[38] B. W. McMenamin, A. J. Shackman, L. L. Greischar, and R. J. Davidson, "Electromyogenic artifacts and electroencephalographic inferences revisited," NeuroImage, vol. 54, no. 1, pp. 4-9, 2011.
[39] M. Zima, P. Tichavský, K. Paul, and V. Krajča, "Robust removal of short-duration artifacts in long neonatal EEG recordings using wavelet-enhanced ICA and adaptive combining of tentative reconstructions," Physiological Measurement, vol. 33, no. 8, pp. N39-N49, 2012.

[40] G. Lio and P. Boulinguez, "Greater robustness of second order statistics than higher order statistics algorithms to distortions of the mixing matrix in blind source separation of human EEG: implications for single-subject and group analyses," NeuroImage, vol. 67, pp. 137-152, 2013.

[41] J. Metsomaa, J. Sarvas, and R. J. Ilmoniemi, "Multi-trial evoked EEG and independent component analysis," Journal of Neuroscience Methods, vol. 228, pp. 15-26, 2014.

[42] M. Chaumon, D. V. M. Bishop, and N. A. Busch, "A practical guide to the selection of independent components of the electroencephalogram for artifact correction," Journal of Neuroscience Methods, vol. 250, pp. 47-63, 2015.

[43] J. A. Urigüen and B. Garcia-Zapirain, "EEG artifact removalstate-of-the-art and guidelines," Journal of Neural Engineering, vol. 12, no. 3, Article ID 031001, 2015.

[44] G. L. Wallstrom, R. E. Kass, A. Miller, J. F. Cohn, and N. A. Fox, "Automatic correction of ocular artifacts in the EEG: a comparison of regression-based and component-based methods," International Journal of Psychophysiology, vol. 53, no. 2, pp. 105119, 2004.

[45] M. Plöchl, J. P. Ossandón, and P. König, "Combining EEG and eye tracking: identification, characterization, and correction of eye movement artifacts in electroencephalographic data," Frontiers in Human Neuroscience, vol. 6, article 278, 2012.

[46] J. M. Zumer, H. T. Attias, K. Sekihara, and S. S. Nagarajan, "A probabilistic algorithm integrating source localization and noise suppression for MEG and EEG data," NeuroImage, vol. 37, no. 1, pp. 102-115, 2007.

[47] J. M. Zumer, H. T. Attias, K. Sekihara, and S. S. Nagarajan, "Probabilistic algorithms for MEG/EEG source reconstruction using temporal basis functions learned from data," NeuroImage, vol. 41, no. 3, pp. 924-940, 2008.

[48] M. A. Uusitalo and R. J. Ilmoniemi, "Signal-space projection method for separating MEG or EEG into components," Medical and Biological Engineering and Computing, vol. 35, no. 2, pp. 135-140, 1997.

[49] R. R. Ramírez, B. H. Kopell, C. R. Butson, B. C. Hiner, and S. Baillet, "Spectral signal space projection algorithm for frequency domain MEG and EEG denoising, whitening, and source imaging," NeuroImage, vol. 56, no. 1, pp. 78-92, 2011.

[50] R. Näätänen, P. Paavilainen, T. Rinne, and K. Alho, “The mismatch negativity (MMN) in basic research of central auditory processing: a review," Clinical Neurophysiology, vol. 118, no. 12, pp. 2544-2590, 2007.

[51] M. I. Garrido, J. M. Kilner, K. E. Stephan, and K. J. Friston, "The mismatch negativity: a review of underlying mechanisms," Clinical Neurophysiology, vol. 120, no. 3, pp. 453-463, 2009.

[52] M. Tervaniemi, M. Huotilainen, and E. Brattico, "Melodic multi-feature paradigm reveals auditory profiles in musicsound encoding," Frontiers in Human Neuroscience, vol. 8, article 496, 2014.

[53] M. Tervaniemi, L. Janhunen, S. Kruck, V. Putkinen, and M. Huotilainen, "Auditory profiles of classical, jazz, and rock musicians: genre-specific sensitivity to musical sound features," Frontiers in Psychology, vol. 6, article 1900, 2016.

[54] V. Putkinen, M. Tervaniemi, K. Saarikivi, N. de Vent, and M. Huotilainen, "Investigating the effects of musical training 
on functional brain development with a novel Melodic MMN paradigm," Neurobiology of Learning and Memory, vol. 110, pp. 8-15, 2014.

[55] A. Gramfort, M. Luessi, E. Larson et al., "MEG and EEG data analysis with MNE-Python," Frontiers in Neuroscience, vol. 7, article 267, 2013.

[56] A. Gramfort, M. Luessi, E. Larson et al., "MNE software for processing MEG and EEG data," NeuroImage, vol. 86, pp. 446460, 2014.

[57] S. Makeig, A. J. Bell, T. Jung, and T. J. Sejnowski, "Independent component analysis of electroencephalographic data," in Advances in Neural Information Processing Systems, pp. 145-151, 1996.

[58] R. Oostenveld, P. Fries, E. Maris, and J.-M. Schoffelen, "FieldTrip: open source software for advanced analysis of MEG, EEG, and invasive electrophysiological data," Computational Intelligence and Neuroscience, vol. 2011, Article ID 156869, 9 pages, 2011.

[59] H. Nolan, R. Whelan, and R. B. Reilly, "FASTER: fully automated statistical thresholding for EEG artifact rejection," Journal of Neuroscience Methods, vol. 192, no. 1, pp. 152-162, 2010.

[60] P. Comon, "Independent component analysis, a new concept?" Signal Processing, vol. 36, no. 3, pp. 287-314, 1994. 

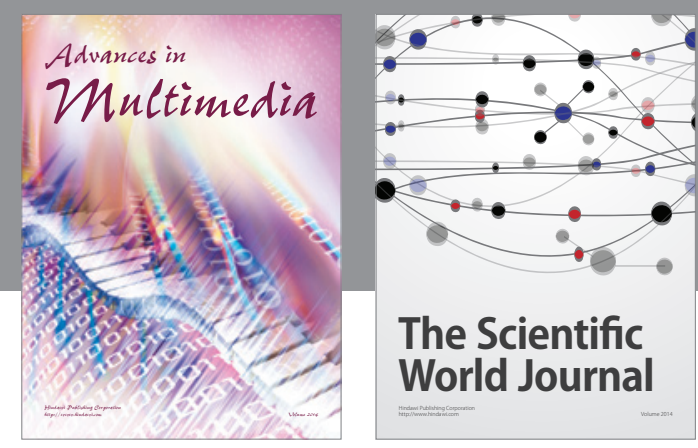

The Scientific World Journal
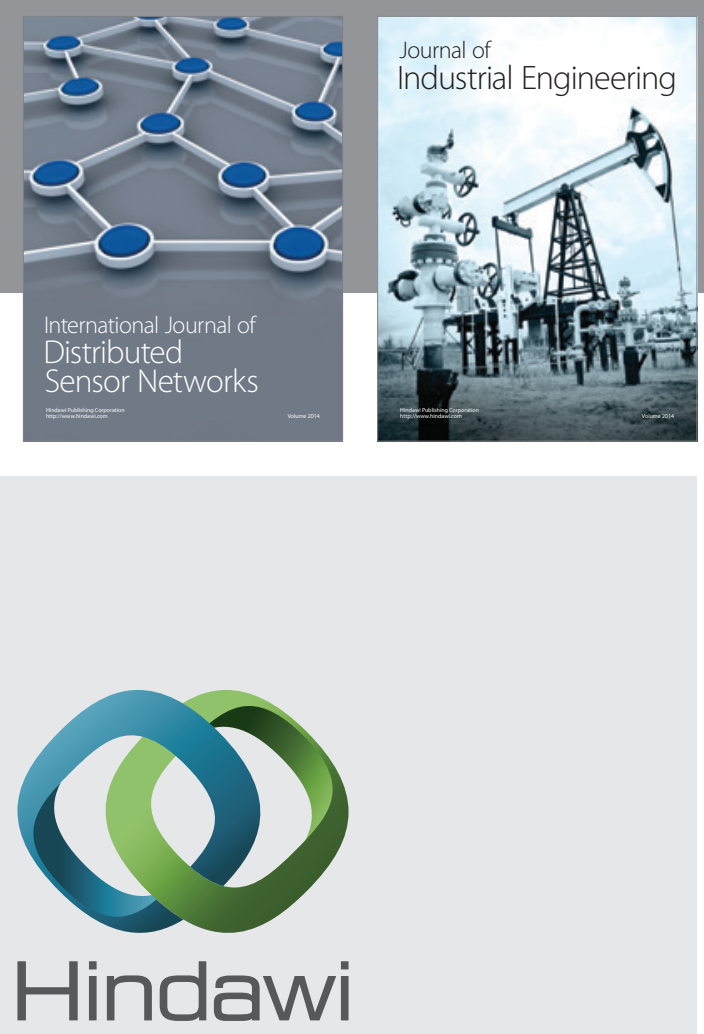

Submit your manuscripts at

http://www.hindawi.com

\section{Computer Networks} and Communications
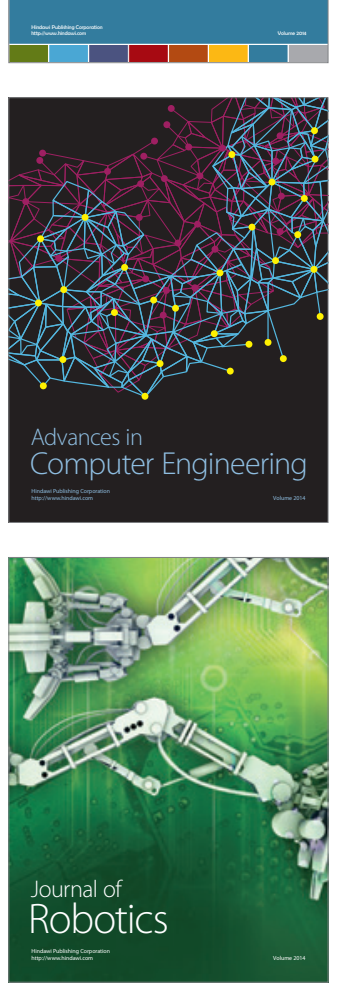
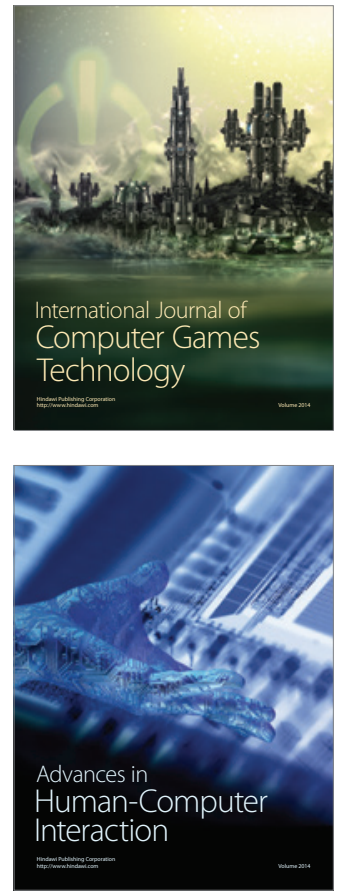
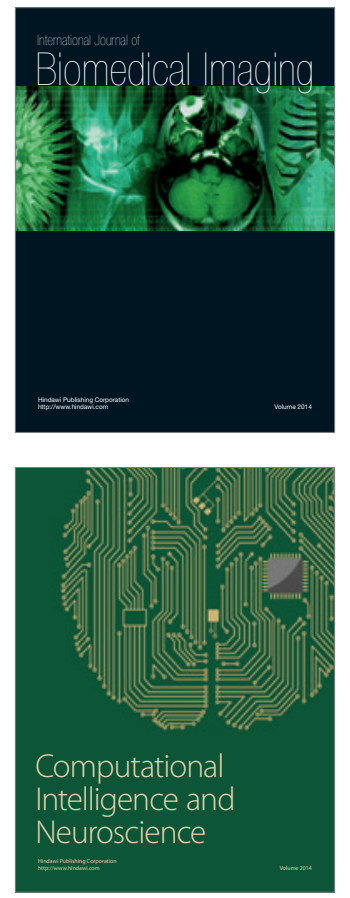
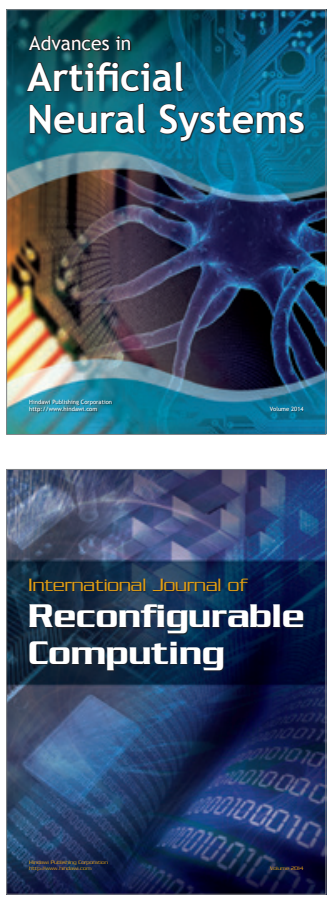
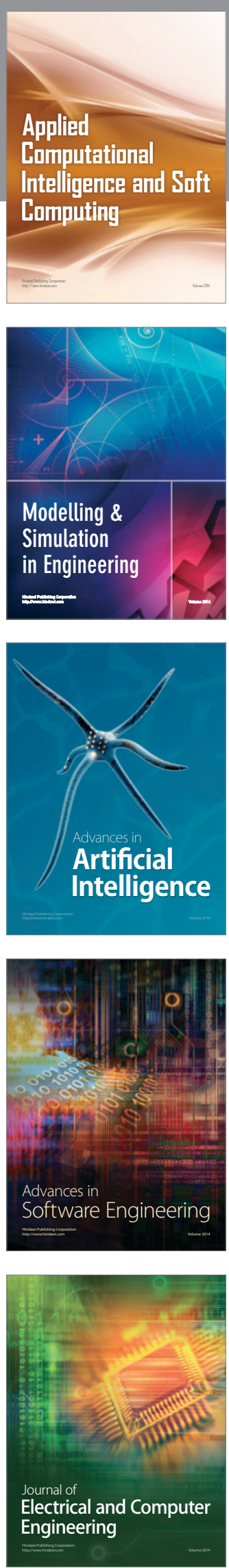